\title{
Activation Energy for Grain Growth of the Isochronally Annealed Ultrafine Grained Magnesium Alloy after Hot Extrusion and Equal-Channel Angular Pressing (EX-ECAP)
}

\author{
J. StrÁSKÁ*, J. StrŔsKÝ AND M. JANEČEK
}

Department of Physics of Materials, Charles University in Prague, Ke Karlovu 5, 12116 Praha 2, Czech Republic

\begin{abstract}
Magnesium alloy AZ31 prepared by hot extrusion and 4 passes of equal-channel angular pressing (EX-ECAP) has ultra-fine grained microstructure with an average grain size of $900 \mathrm{~nm}$. Grain growth is analysed using a general equation for the grain growth and an Arrhenius equation. The calculated value of the activation energy for grain growth differs with the annealing temperature. The fitted value of activation energy for grain growth in the intermediate temperature range $\left(210-400^{\circ} \mathrm{C}\right)$ is in accordance with the results of other authors, but it is shown in this study that such value is abnormally low and physically meaningless. More real values of apparent activation energy in this temperature range were calculated from the model assuming a linear increase of activation energy with increasing annealing temperature. Result of this linear model of evolution of activation energy in the temperature range between $210-400^{\circ} \mathrm{C}$ is expressed by the interval estimation of apparent activation energy values. It is concluded that the evolution of apparent activation energy can be explained by a change in the mechanism underlying the grain boundary migration. In the low temperature range, the grain boundary diffusion is dominant since the material is ultra-fine grained, whereas at higher temperatures, the lattice self-diffusion is more important.
\end{abstract}

DOI: $10.12693 /$ APhysPolA.128.578

PACS: 62.20.fq, 81.05.Bx, 81.20.Hy, 81.40.Lm, 81.70.Bt

\section{Introduction}

Ultra-fine grained (UFG) metals processed by severe plastic deformation (SPD) methods are very promising materials for applications in automotive, aerospace or biomedical industries. Equal-channel angular pressing (ECAP) is one of the most commonly used SPD technique. Repetition of ECAP can produce fully-dense bulk solid with UFG microstructure and high fraction of highangle grain boundaries [1]. Experiments suggest that ECAP is most effective in magnesium-based alloys when used after extrusion. This two-step process consisting of hot extrusion followed by several ECAP passes is designated EX-ECAP and was used effectively with a $\mathrm{Mg}-\mathrm{Zr}$ alloy [2] and subsequently with $\mathrm{Mg}-\mathrm{Al}[3]$ and $\mathrm{Mg}-\mathrm{Al}-$ Zn [4] alloys. The magnesium alloy AZ31, investigated in this study, is one of the most commonly used magnesium alloy in the industry [5]. Various properties (e.g. mechanical properties, corrosion resistance or microstructure) of this alloy are enhanced after the EX-ECAP technique which was also studied in detail and published in our previous papers [6-9].

Microstructure stability of the fine-grained AZ31 alloy was investigated by many authors, e.g. Chao et al. [10] or Yang et al. Particularly, Kim [11], Kim and Kim [12] and Radi and Mahmudi [13] focused on microstructure stability of AZ31 after ECAP. All three papers present calculations of activation energies for grain growth and identify two or three temperature regimes with significantly different values of activation energy. Suspiciously low value

*corresponding author; e-mail: straska.jitka@gmail.com of activation energy in some temperature ranges is observed but not fully explained. Lian et al. [14] assume a continuous increase of activation energy $Q$ to obtain a reasonable fit using the standard equation for grain growth. It is assumed that $Q$ is rising due to nonequilibrium nature of grain boundaries.

The distinct feature of ultrafine grained materials is, naturally, a high amount of grain boundaries. The grain boundary diffusion therefore plays a major role in grain growth in these materials $[11,12,14,15]$. The activation energy of the grain boundary diffusion is therefore an important parameter of grain growth kinetics. Moreover, some authors claim that the special nature of grain boundaries in UFG materials - non-equilibrium grain boundaries - can significantly decrease the activation energy associated with grain growth [15]. The decrease of amount of grain boundaries, which is naturally associated with the grain growth, causes the lattice selfdiffusion to become the dominant process at the expense of grain boundary diffusion. This effect leads to a change of the apparent activation energy at different temperature ranges which is determined from the Arrhenius equation [16]. This fact is indirectly mentioned in [14], but completely omitted in other papers dealing with the determination of activation energy associated to grain growth (e.g. $[11,12,15])$. In this paper, we assume that the dominant process responsible for grain growth continuously shifts from grain boundary diffusion to lattice self-diffusion. This approach is able to explain unusually low activation energies reported by other authors who ascribed this fact to non-equilibrium grain boundaries without a clear proof (e.g. [15]). This contribution follows our previous experimental study of microstructure 
stability at elevated temperature [17]. Experimental results are not repeated here due to length limit of the paper.

\section{Experimental material and procedures}

As-cast commercial AZ31 alloy (nominal composition $\mathrm{Mg}-3 \% \mathrm{Al}-1 \% \mathrm{Zn}$ ) was firstly extruded at $350^{\circ} \mathrm{C}$ with an extrusion ratio of 22 and secondly processed by 4 passes of ECAP with outer angle $90^{\circ}$ at $180^{\circ} \mathrm{C}$ using route $B_{c}$. More details can be found in [17]. Series of specimens for thermal-stability investigation were prepared by isochronal annealing at temperatures $150-500^{\circ} \mathrm{C}$ for $1 \mathrm{~h}$ followed by water-quench.

Microstructure observations were published in our previous paper [17].

\section{Results and discussion}

Process of grain growth during isothermal annealing could be analysed using a general equation for grain growth and an Arrhenius equation

$$
d^{n}-d_{0}^{n}=t k=t k_{0} \exp \left(-\frac{Q_{\mathrm{g}}}{R T}\right),
$$

where $d$ is the grain size at given annealing time, $d_{0}$ is the initial grain size, $n$ is the grain growth exponent, $t$ is the annealing time, $k_{0}$ is a constant, $Q_{\mathrm{g}}$ is the activation energy, $R$ is the gas constant and $T$ is the absolute temperature. The annealing time $t$ is equal to $1 \mathrm{~h}$ and is constant for all measurements. Note that the main assumption of the model is that the evolution of grain size with temperature is fully captured by the exponential function. Terms $k_{0}$ and $Q_{\mathrm{g}}$ are assumed to be temperature independent. Otherwise the model is not valid and the fitted parameters do not represent any physical value.

In our study, we use $n=2$ for most computations. This value follows from the standard model of normal grain growth and grain boundary migration [18]. However, it is uneasy to unambiguously support this choice since isothermal annealing was not performed. Previous studies $[12,19,20]$ reported a value of $n$ in a range from 2 to 8 for various magnesium alloys and magnesiumbased composites. The value of grain growth exponent $n$ observed in ultrafine-grained magnesium alloy AZ31 produced by various techniques of severe plastic deformation ranges between 2 and $4[10,12,21]$. It is moreover explicitly or implicitly assumed that the nature of the process (the values $k_{0}$ and $Q_{\mathrm{g}}$ ) remain constant during isothermal annealing. Note that when this assumption is not fulfilled, the value of $n$ is overestimated. The AZ31 alloy processed by the most similar process was studied by Kim et al. [11, 12] and they used the grain growth exponent $n$ equal to 2 . We computed the values of activation energy for $n=2,3$ and 4 (see Fig. 1), but for further computations we use the value $n=2$, which will allow us to make comparison with the most related papers.

The activation energy $Q_{\mathrm{g}}$ could be determined as the slope of the dependence of $\ln \left(d^{n}-d_{0}^{n}\right)$ on $1 / T$ which is shown in Fig. 1 for $n=2,3$ and 4 . Three temperature ranges with different $Q_{\mathrm{g}}$ values can be distin-

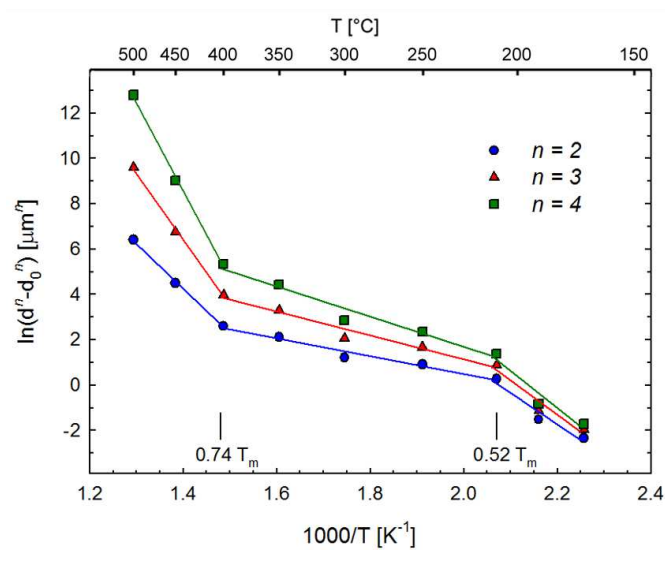

Fig. 1. Plot of $\ln \left(d^{n}-d_{0}^{n}\right)$ vs. $1000 / T$ for the estimation of the activation energy for grain growth of the EX-ECAP magnesium alloy AZ31.

guished. The calculated values of activation energy according to Eq. (1) are summarized in Table I along with reliability of the fit in selected temperature ranges for different choices of parameter $n$.

TABLE I

Calculated values of activation energy $Q_{\mathrm{g}}$ and reliability of the fit for three different temperature ranges and grain growth exponent $n=2,3$ and 4 .

\begin{tabular}{c|c|c|c}
\hline \hline$T\left[{ }^{\circ} \mathrm{C}\right]$ & $170-210{ }^{\circ} \mathrm{C}$ & $210-400{ }^{\circ} \mathrm{C}$ & $400-500{ }^{\circ} \mathrm{C}$ \\
\hline$n=2$ & $115 \mathrm{~kJ} / \mathrm{mol}$ & $33 \mathrm{~kJ} / \mathrm{mol}$ & $164 \mathrm{~kJ} / \mathrm{mol}$ \\
& $(R=0.945)$ & $(R=0.971)$ & $(R=0.998)$ \\
\hline \multirow{2}{*}{$n=3$} & $126 \mathrm{~kJ} / \mathrm{mol}$ & $44 \mathrm{~kJ} / \mathrm{mol}$ & $242 \mathrm{~kJ} / \mathrm{mol}$ \\
& $(R=0.937)$ & $(R=0.966)$ & $(R=0.998)$ \\
\hline \multirow{2}{*}{$n=4$} & $137 \mathrm{~kJ} / \mathrm{mol}$ & $56 \mathrm{~kJ} / \mathrm{mol}$ & $322 \mathrm{~kJ} / \mathrm{mol}$ \\
& $(R=0.929)$ & $(R=0.962)$ & $(R=0.998)$
\end{tabular}

The activation energy $Q_{\mathrm{g}}$ in the high temperature range $\left(T>400{ }^{\circ} \mathrm{C}\right)$ shall be related to the lattice selfdiffusion in pure magnesium $(135 \mathrm{~kJ} / \mathrm{mol}[22])$ since the lattice self-diffusion is the dominant process of grain growth when the material consists already of comparatively bigger grains. The fit using $n=2$ provides a value of $164 \mathrm{~kJ} / \mathrm{mol}$, which might be substantiated for AZ31 alloy. Fitting using $n=2$ and $n=3$ seem to highly overestimate the true value of activation energy of lattice self-diffusion and therefore we will consider $n=2$ for the rest of the paper. Kim and Kim [12] and Radi and Mahmudi [13] determined the activation energy in the same high-temperature range as 114 and $94 \mathrm{~kJ} / \mathrm{mol}$, respectively, which are again lower values of $Q_{\mathrm{g}}$ comparing to ours.

The fitted activation energy $Q_{\mathrm{g}}$ in the low temperature range $\left(T>210^{\circ} \mathrm{C}\right)$ is equal to $115 \mathrm{~kJ} / \mathrm{mol}$, which is higher than the activation energy for grain boundary diffusion in pure magnesium $(92 \mathrm{~kJ} / \mathrm{mol}[22])$. The activation energy of grain boundary diffusion in alloys with thermally stable phases is generally higher than the activation energy of GB diffusion in pure metals [13] and our results are in agreement with this fact. Kim and 
Kim evaluated the activation energy in the similar temperature range $\left(200-250^{\circ} \mathrm{C}\right)$ and they report the value of $70 \mathrm{~kJ} / \mathrm{mol}$ [12]. A similar value of activation energy, $74 \mathrm{~kJ} / \mathrm{mol}$, was also calculated by Radi and Mahmudi at temperatures $217-257^{\circ} \mathrm{C}[13]$. These values are significantly lower than in our study, but their starting value of $^{\prime} d_{0}$ is equal to $2.5 \mu \mathrm{m}$ and $6.4 \mu \mathrm{m}$ in [12] and [13], respectively, due to different material processing compared to our study [17] where $d_{0}$ is $0.9 \mu \mathrm{m}$ and even after annealing at $250{ }^{\circ} \mathrm{C}$ the grain size does not reach $2 \mu \mathrm{m}$. We therefore argue that by far the most dominant process of the grain growth in our study at low temperatures is the grain boundary diffusion.

It is shown in Kim and Kim [12] and supported by our results that the low value of activation energy in the intermediate temperature range $210-400{ }^{\circ} \mathrm{C}$ cannot be substantiated. It is argued in [12] that the diffusivity that is the driving force for grain growth is changing due to recovery process and then the Arrhenius equation (Eq. (1)) is not valid making results of the fit meaningless. Kim and Kim [12] provide the hypothesis that abnormally low $Q_{\mathrm{g}}$ is caused by a change of dislocation density that alters parameter $k_{0}$ due to pipe diffusion. We agree that the value of $Q_{\mathrm{g}}$ arising from the fit is physically meaningless, but we disagree that this is caused by changing the factor $k_{0}$. Instead, it is shown that the results can be fully explained by assuming temperature dependent apparent activation energy $Q_{\mathrm{g}}$.

A detailed look at the results of the fit shows that the coefficient $k_{0}$ (see Table II) is actually smaller for lower temperatures. This is contradictory to the conclusions of [12], because the diffusivity at lower temperatures should be bigger due to dislocations providing pipe diffusion.

TABLE II

Fitted values of $k_{0}$ for three temperature ranges.

\begin{tabular}{c|c}
\hline \hline Temperature $\left[{ }^{\circ} \mathrm{C}\right]$ & $k_{0}\left[\mu \mathrm{m}^{2} \mathrm{~h}^{-1}\right]$ \\
\hline $170-210$ & $3.1 \times 10^{12}$ \\
$210-400$ & $4.4 \times 10^{3}$ \\
$400-500$ & $67.3 \times 10^{12}$
\end{tabular}

TABLE III

Values of decisive term $k_{0} \exp \left(-Q_{\mathrm{g}}\right)$ for three temperature ranges.

\begin{tabular}{c|c}
\hline \hline Temperature $\left[{ }^{\circ} \mathrm{C}\right]$ & $\boldsymbol{k}_{0} \exp \left(-Q_{\mathrm{g}}\right)\left[\frac{\mathrm{kJ} \mu \mathrm{m}^{2}}{\mathrm{molh}}\right]$ \\
\hline $170-210$ & $2.9 \times 10^{-38}$ \\
$210-400$ & $2.6 \times 10^{-11}$ \\
$400-500$ & $4.7 \times 10^{-58}$
\end{tabular}

On the other hand, the activation energy is higher for higher temperatures, which confirms the fact that diffusivity is lower, when grain boundary diffusion is less important than the lattice self-diffusion. It can be easily shown that the effect of changing apparent $Q_{\mathrm{g}}$ is much stronger than the effect of changing the factor $k_{0}$ by comparing the decisive term $k_{0} \exp \left(-Q_{\mathrm{g}}\right)$ (see Table III).
Higher diffusivity coefficient at lower temperatures is therefore captured by the lower activation energy for grain growth $Q_{\mathrm{g}}$, whereas the effect of $k_{0}$ is low.

We argue that the abnormally low value of fitted activation energy in the intermediate region is neither the activation energy of some new process (e.g. grain boundary diffusion of non-equilibrium boundaries) nor the result of changing pre-multiplier $k_{0}$. It is considered that the fitted value of the $Q_{\mathrm{g}}$ is wrong since it is based on the wrong assumption that $Q_{\mathrm{g}}$ is constant over the whole temperature range. On the other hand, we assume that the apparent activation energy is continuously changing from the activation energy of the grain boundary diffusion to the activation energy of the lattice self-diffusion.

In the following, we prove by simple model that this assumption is fully supported by the experimental data. Let us assume that in the transition temperature range the apparent activation energy $Q_{\mathrm{g}}$ increases linearly

$$
Q_{\mathrm{g}}=c_{0}+c_{1} T \text {, }
$$
where $0<c_{0}<Q_{\mathrm{g}}$ and $c_{1}>0$ are constants.

This is indeed a simplification, but this model is sufficient to explain the evolution of the apparent activation energy. Let us substitute Eq. (2) to the Arrhenius equation

$$
\ln \left(d^{2}-d_{0}^{2}\right)=\ln \left(k_{0}\right)-\frac{c_{0}+c_{1} T}{R T} .
$$

This can be simply rewritten to the form that is used for fitting as shown in Eq. (1). After reorganization we get

$$
\ln \left(d^{2}-d_{0}^{2}\right)=\ln \left(k_{0}\right)-\frac{c_{1}}{R}-\frac{c_{0}}{R} \frac{1}{T} .
$$

It is clear that the intercept of the fit (fitted $\ln \left(k_{0}\right)$ when $Q_{\mathrm{g}}$ assumed constant) equals to $\ln \left(k_{0}\right)-\frac{c_{1}}{R}$ (when $Q_{\mathrm{g}}$ assumed linearly rising), which is obviously a lower number just due to assumption of linearly increasing apparent $Q_{\mathrm{g}}$. At the same time, the slope of the fit (fitted $\left.\frac{Q_{\mathrm{g}}}{R}\right)$ equals to $\frac{c_{0}}{R}$, which is again much lower number than "true" $\frac{Q_{\mathrm{g}}}{R}$. Therefore both apparent $k_{0}$ and fitted $Q_{\mathrm{g}}$ from the fit in transition region are not connected to any physical process, despite the fact that fitting by the Arrhenius equation is successful (the error of the fit is low).

According to the model, the values of $c_{0}, c_{1}$ and $k_{0}$ shall be fitted. The coefficient $c_{0}$ should correspond to the fitted $Q_{\mathrm{g}}$ from the fit of the transition region (see Fig. 1). The coefficient $c_{1}$ can be then computed from linear dependence of $Q_{\mathrm{g}}$ on the temperature using Eq. (2) for different values of $Q_{\mathrm{g}}$ and $T$. However, values of the activation energy of grain boundary diffusion and the activation energy of lattice self-diffusion correspond to the temperature intervals of $170-210^{\circ} \mathrm{C}$ and $400-500{ }^{\circ} \mathrm{C}$, respectively. Therefore, only an interval estimate of $c_{1}$ can be obtained. Interval of the values of apparent activation energy $Q_{\mathrm{g}}$ calculated from the minimal and maximal values of $c_{1}$ and $c_{0}$ that equals the fitted $Q_{\mathrm{g}}$ in the intermediate region is displayed in Fig. 2 along with activation energies that are attributed to grain boundary diffusion (low temperature region) and lattice self-diffusion (high temperature region). 


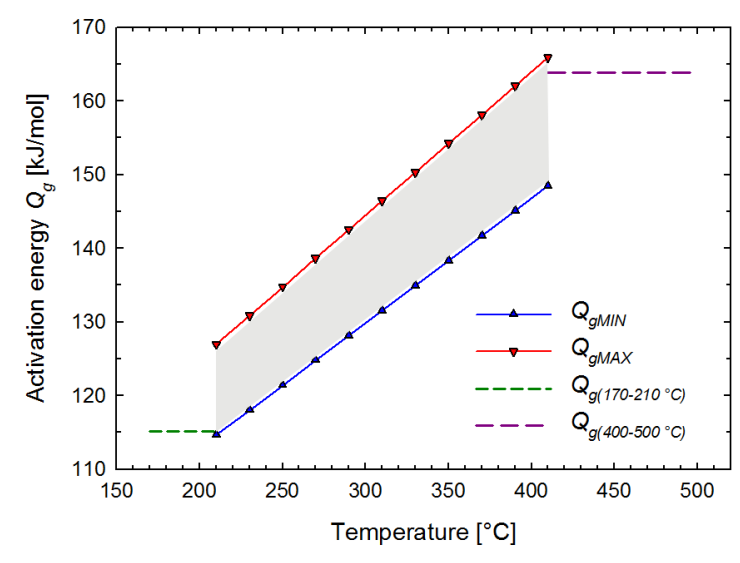

Fig. 2. Plot of the fitted values of the activation energy for grain growth $Q_{\mathrm{g}}$ for three temperature ranges; $Q_{\mathrm{g}}$ in the intermediate temperature range is defined by the interval between minimal and maximal $Q_{\mathrm{g}}$ (grey area).

The low values of activation energy in the intermediate temperature region reported by $[11,12,15]$ follow from misinterpretation of the model of grain growth. In this study, it is assumed that grain growth is dominated by grain boundary diffusion for lower temperatures until the grains are sufficiently small followed by the lattice selfdiffusion process for higher temperatures when grains are already larger. The continuous change of apparent activation energy can be modeled by taking $Q_{\mathrm{g}}$ as a linear function of $T$. The experimental data fully support such model. The low values of $Q_{\mathrm{g}}$ reported by other authors (e.g. [12]) can be fully explained by a continuous change of apparent activation energy from the grain boundary diffusion process to the lattice self-diffusion.

\section{Conclusions}

The microstructure stability of ultrafine-grained magnesium alloy AZ31 was studied in the temperature range $150-500^{\circ} \mathrm{C}$. The process of grain growth was analysed using a general equation for grain growth and an Arrhenius equation. The activation energies were determined for different temperature ranges.

It is shown that the activation energy calculated from standard Arrhenius equation assuming constant activation energy is physically meaningless in the transition temperature range.

We assumed a linear increase with temperature of the apparent activation energy in this transition temperature range and computed the interval estimate of the apparent activation energy that is consistent with values in higher and lower temperature regions and also with the values calculated by a simple Arrhenius fit. The grain growth in AZ31 alloy processed by ECAP can be fully explained by a continuous transition in dominant diffusion processes from the grain boundary diffusion to the lattice self-diffusion.

\section{Acknowledgments}

This study was financially supported by GACR under the Grant P107/13/13616S. J. Stráská acknowledges the financial support by student grants GAUK 530712/2012 and SVV 2014-267303.

\section{References}

[1] R.Z. Valiev, T.G. Langdon, Prog. Mater. Sci. 51, 881 (2006).

[2] Z. Horita, K. Matsubara, K. Makii, T.G. Langdon, Scr. Mater. 47, 255 (2002).

[3] K. Matsubara, Y. Miyahara, Z. Horita, T.G. Langdon, Acta Mater. 51, 3073 (2003).

[4] Y. Miyahara, Z. Horita, T.G. Langdon, Mater. Sci. Eng. A 420, 240 (2006).

[5] H.E. Friedrich, B.L. Mordike, Magnesium Technology: Metallurgy, Design Data, Automotive Applications, Springer Science \& Business Media, Berlin 2006.

[6] M. Janeček, S. Yi, R. Král, J. Vrátná, K.U. Kainer, J. Mater. Sci. 45, 4665 (2010).

[7] M. Janeček, J. Čížek, J. Gubicza, J. Vrátná, J. Mater. Sci. 47, 7860 (2012).

[8] J. Vrátná, M. Janeček, J. Čížek, D.J. Lee, E.Y. Yoon, H.S. Kim, J. Mater. Sci. 48, 4705 (2013).

[9] J. Vrátná, B. Hadzima, M. Bukovina, M. Janeček, J. Mater. Sci. 48, 4510 (2013).

[10] H.Y. Chao, H.F. Sun, W.Z. Chen, E.D. Wang, Mater. Charact. 62, 312 (2011).

[11] H.K. Kim, J. Mater. Sci. 39, 7107 (2004).

[12] H.K. Kim, W.J. Kim, Mater. Sci. Eng. A 385, 300 (2004).

[13] Y. Radi, R. Mahmudi, Mater. Sci. Eng. A 527, 2764 (2010).

[14] J. Lian, R.Z. Valiev, B. Baudelet, Acta Metall. Mater. 43, 4165 (1995).

[15] J. Wang, Y. Iwahashi, Z. Horita, M. Furukawa, M. Nemoto, R.Z. Valiev, T.G. Langdon, Acta Mater. 44, 2973 (1996).

[16] F.G. Helfferich, Kinetics of Multistep Reactions, Elsevier 2004.

[17] J. Stráská, M. Janeček, J. Čížek, J. Stráský, B. Hadzima, Mater. Charact. 94, 69 (2014).

[18] G. Gottstein, Physical Foundations of Materials Science, Springer, Berlin 2004.

[19] P. Cao, L. Lu, M.O. Lai, Mater. Res. Bull. 36, 981 (2001).

[20] M.A. Thein, L. Lu, M.O. Lai, Compos. Sci. Technol. 66, 531 (2006).

[21] Q. Miao, L. Hu, X. Wang, E. Wang, J. Alloys Comp. 493, 87 (2010).

[22] H.J. Frost, M.F. Ashby, Deformation-Mechanism Maps: The Plasticity and Creep of Metals and Ceramics, Pergamon Press, New York 1982. 